\title{
Disease profiling by MALDI MS analysis of biofluids
}

Stephane Camuzeaux and John F. Timms ${ }^{\dagger}$

Cancer Proteomics Laboratory, Institute for Women's Health, University College London, UK

†Corresponding author email: john.timms@ucl.ac.uk 


\section{Abstract}

There is an urgent need for accurate biomarkers of disease. The low-molecular weight proteome of blood serum or other biological fluids may be an ideal source of such biomarkers, although its analysis requires high-throughput strategies to enrich and quantify peptides and small proteins with biomarker potential. Herein, serum samples from cancer cases and controls are compared using a workflow of robotic reversed-phase extraction and clean-up, followed by automated MALDI MS spectral acquisition and analysis of the low-molecular weight peptidome. The aim of the presented methodology is to facilitate the discovery of candidate serum biomarkers of cancer using MALDI MS profiling, although the method is applicable to any comparative proteomic analysis of any biofluid.

\section{Key words}

MALDI MS, proteomic profiling, MS profiling, serum, peptidome, LC-MS/MS, blood, biofluids, sample handling 


\section{Introduction}

There is an urgent need for accurate disease biomarkers, which may provide novel approaches to diagnosis and screening. The blood serum/plasma proteome may be an ideal source of such biomarkers, although its complexity necessitates novel strategies to enrich and quantify low-abundance protein species with biomarker potential. The low-molecular weight proteome or 'peptidome' of serum/plasma is also a source of potential disease biomarkers (Geho et al. 2006; Hortin 2006; Liotta and Petricoin 2006; Petricoin et al. 2006; Villanueva et al. 2006a). Low-molecular weight proteins and peptides occur endogenously within the bloodstream, whilst protein fragments may be derived in vivo or ex vivo through the action of proteases on both blood-borne and cell/tissue-derived proteins. Both MALDI and its derivative SELDI MS are well-suited for profiling the peptidome of biofluids and are particularly suited for high-throughput analyses, i.e. profiling of 100 s or 1000 s of samples in a single experiment. For comparative analyses in biomarker discovery, peptide peak intensities (peak areas or heights) from spectra acquired from multiple samples are compared between different clinical and control groups using dedicated analysis software tools. Differences in peak intensities are typically reported as ratios between the groups with an associated statistic to evaluate the significance of any differences. Since the comparisons are multivariate, correction for multiple testing must be applied. Any peaks of interest must then be identified, usually using orthogonal methods such as tandem MS or antibody-based methods. The onus is on proving that a peak identified by such methods is exactly the same species as the differential peak identified from the (MALDI) MS profiling. This is not always trivial, particularly given the lower mass accuracy of typical MALDI time-of-flight (TOF) instruments. Ideally, tandem MS identification is performed within the same experiment as the profiling.

Much criticism has been levelled at low-molecular weight serum/plasma profiling for biomarker discovery using MALDI and SELDI MS (Diamandis 2004a, 2006; Davis et al. 2010). This largely concerns experimental bias introduced during preanalytical sample handling. It is without doubt the case that proteolysis has a huge 
influence on the peptidome patterns of biofluids, particularly serum. Any difference in handling (e.g. clotting time, temperature, storage etc.) between samples may affect proteolysis and has been shown to influence the resulting peptidome patterns (Karsan et al. 2005; Banks et al. 2005; Timms et al. 2007; Baggerly et al. 2004). Essentially, this gives rise to technical variation that can mask true biological variation and increase the false positive rate. Thus, in any profiling study it must be ensured that all samples are collected, handled and stored as identically as possible to avoid such bias. Concerns have also been raised over assay reproducibility and the robustness of class-discriminating algorithms used for MALDI MS profiling biomarker discovery (Diamandis 2004b; Baggerly et al. 2005). Thus, monitoring and reporting of platform reproducibility is obligatory, whilst the robustness of algorithms must be assessed through proper training and test set validation. Finally, it has been argued that these high-throughput methods lack the sensitivity to detect lowabundance species, with coverage limited to abundant small proteins and fragments of coagulation proteins. This appears to be the case as evidenced by identifications assigned to MALDI MS spectral peaks recorded from serum samples, where fibrinogen fragments were the predominant species (Tiss et al. 2010). However, this is not to say that these small proteins, endogenous peptides or proteolytic fragments do not have potential as disease biomarkers as many seemingly well-controlled studies have reported. Indeed, it has been proposed that fragments of abundant coagulation proteins are surrogate peptide markers of cancer, generated ex vivo during coagulation through the action of tumour-specific exopeptidases (Villanueva et al. 2006c). This may also hold true in other diseases, where low-abundance, disease-associated proteases may generate specific patterns of protein fragments with diagnostic potential. 


\section{Applications}

Herein, we describe a reproducible, high-throughput, semi-automated, MALDI MS profiling method similar to that used in (Timms et al. 2010) (see Figure 1). The method describes the profiling of serum from case control sample sets, although it is equally applicable to other biological fluids/clinical specimens such as plasma, cerebrospinal fluid, urine, ascites and saliva. We also describe the data analysis steps and a protocol for identification of discriminatory peaks with biomarker potential. The aim of the presented methodology is to facilitate the discovery of candidate serum biomarkers for the differential diagnosis of ovarian cancer.

This protocol and a slight variation of it with regard to the serum peptide extraction step (Tiss et al. 2007) have been used for biomarker discovery studies for the early detection of ovarian cancer (Timms et al. 2010; Timms et al. 2011) and differential diagnosis of biliary tract cancer (Sandanayake et al. 2014). Similar protocols have been used by other groups for diagnostic biomarker discovery in a variety of cancers (Villanueva et al. 2006b; Villanueva et al. 2006c; Pietrowska et al. 2009) and other diseases/infections (Conraux et al. 2013; Li et al. 2012; Terracciano et al. 2011; Teunissen et al. 2011; Xiao et al. 2011), for predictive and prognostic biomarker discovery (Vafadar-Isfahani et al. 2010; Taguchi et al. 2007), and to identify blood-borne markers of ageing (Lu et al. 2012). 


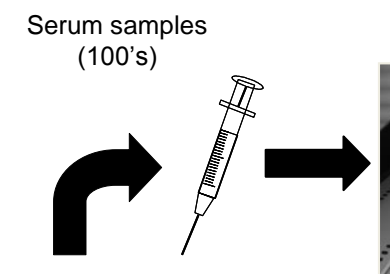

Robotic liquid handling

C18-magnetic beads

Patients/controls

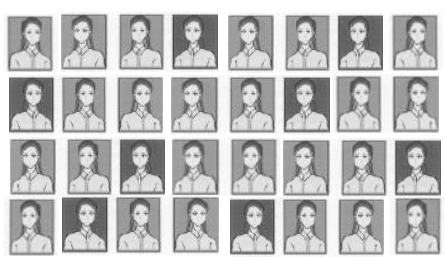

TECAN Genesis
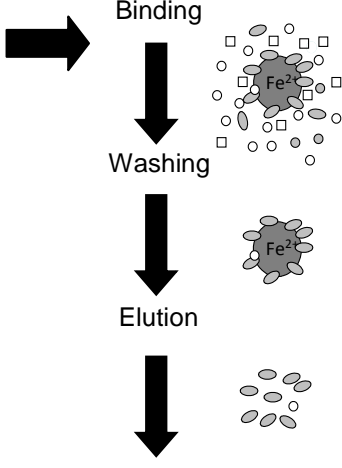

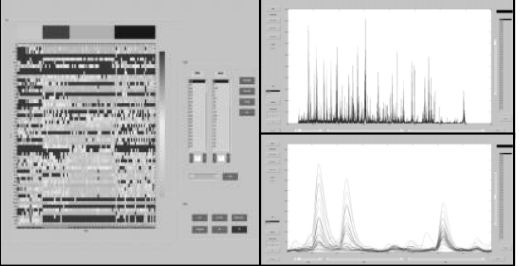

Data pre-processing and Statistical Analysis (ClinProTools/MatLab)

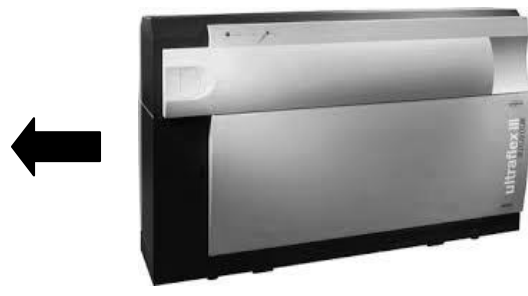

Ultraflex MALDI-TOF Auto-acquisition

Figure 1: Schematic workflow for MALDI MS profiling of biofluids 


\section{Materials and Protocols}

\subsection{Materials}

\subsubsection{Serum collection and storage}

1. 8.5-mL BD Vacutainer ${ }^{\circledR} S S T^{\mathrm{TM}}$ Advance Tubes (Becton Dickinson Diagnostics, New Jersey, US).

2. Bench top centrifuge.

3. Cryovials.

4. $-80^{\circ} \mathrm{C}$ freezer.

5. Quality control human serum (cat. no.: H4522; Sigma-Aldrich Company Ltd, Dorset, UK).

\subsubsection{Serum polypeptide extraction and sample preparation}

1. 96-well Star PCR raised rim skirted plates (Starlab UK Ltd, Milton Keynes, UK).

2. RPC18 Dynabeads (Invitrogen Ltd, Paisley, UK) ${ }^{1}$.

3. 96-well magnetic bead separator (Bruker UK, Coventry, UK).

4. Trifluoroacetic acid (TFA; 100\% HPLC grade; Rathburn Chemicals Ltd, Walkerburn, Scotland).

5. Acetonitrile (ACN; $100 \%$ HPLC grade; Rathburn Chemicals Ltd).

6. Methanol.

7. Genesis Freedom 200 liquid-handling work station (Tecan UK Ltd, Reading, $\mathrm{UK})$.

8. a-Cyano-4-hydroxycinnamic acid (CHCA) matrix solution $(6.2 \mathrm{mg} / \mathrm{mL}$ in 36\%/56\%/8\% methanol/ACN/water; Agilent Technologies UK Ltd, Stockport, UK).

9. Ground steel MALDI target plates (Bruker UK).

\footnotetext{
${ }^{1}$ RPC18 Dynabeads are paramagnetic, non-porous particles modified with hydrophobic $\mathrm{C} 18$ reversed phase chromatographic material. Other manufacturers and chromatographic materials can be used (e.g. C8 reversed phase, weak cation exchange, $\mathrm{Cu}^{2+}-\mathrm{IMAC}$ ), but we have found that the RPC18 Dynabeads gave good yields in terms of the numbers and intensities of the peaks detected.
} 


\subsubsection{MALDI-TOF MS data acquisition and analysis ${ }^{2}$}

1. Bruker Ultraflex II MALDI-TOF/TOF mass spectrometer (Bruker UK).

2. Flexcontrol v2.0 software (Bruker UK) ${ }^{3}$.

3. Peptide calibrant standard II and protein calibrant standard I (Bruker UK).

4. ClinProTools v3.0 software (Bruker UK).

\subsubsection{Peptide Identification by LC-MS/MS}

1. Ultimate 3000 nano $L C$ system coupled to an $L T Q$ Orbitrap $X L$ mass spectrometer (Thermo Fisher Scientific, Hemel Hempstead, UK) via a PicoView nanospray source (New Objective Inc., Woburn, MA, USA). ${ }^{4}$

2. C18 PepMap guard column ( $300 \mu \mathrm{m}$ i.d. $\times 5 \mathrm{~mm}, 5 \mu \mathrm{m}$ bead size, $100 \AA$ pore size; LC Packings, Amsterdam, The Netherlands).

3. C18 PepMap nano LC column $(75 \mu \mathrm{m}$ i.d. $x 150 \mathrm{~mm}, 3 \mu \mathrm{m}$ bead size, $100 \AA$ pore size; LC Packings).

4. Solvent $A: 0.1 \%$ formic acid $(F A)$ in HPLC grade water.

5. Solvent B: $0.1 \%$ FA, $99.9 \%$ HPLC grade ACN (Rathburn Chemicals Ltd).

6. Xcalibur v2.0 (Thermo Scientific) and Mascot Server v2.4 (Matrix Science Ltd, London, UK) software.

7. Nove ${ }^{\circledR}$ Bolt $^{\mathrm{TM}}$ Gel Electrophoresis System with pre-cast $10-20 \%$ gradient SDS-PAGE mini gels and running buffer (Life Technologies, Paisley, UK).

8. InstantBlue colloidal Coomassie blue protein stain (Expedeon, Cambridge, UK).

9. Siliconized Eppendorf tubes.

10. SpeedVac.

\subsection{Methods}

\subsubsection{Serum collection}

\footnotetext{
${ }^{2}$ Other high-performance MALDI-TOF MS systems should be perfectly adequate, though might require additional data analysis software (cf. 3.2.3./3.2.4.).

${ }^{3}$ Software versions higher (newer) than stated should also be adequate.

${ }^{4}$ Other instrumentation and modes of operation can be used for peptide identification.
} 
1. Collect blood samples from consenting subjects by venepuncture into $8.5-\mathrm{mL}$ BD Vacutainer ${ }^{\circledR}$ SST TM $^{\mathrm{TM}}$ Advance tubes. ${ }^{5}$

2. Gently invert tubes 5 times and allow the blood to clot at room temperature for $60 \mathrm{~min}$.

3. Centrifuge tubes at $2,200 \mathrm{rpm}$ at $4^{\circ} \mathrm{C}$ for $10 \mathrm{~min}$ and aliquot $200 \mu \mathrm{L}$ of serum supernatant into clearly labelled cryovials and freeze at $-80^{\circ} \mathrm{C}$ until further use. Record date of sampling and freezing.

\subsubsection{Serum polypeptide extraction and sample preparation}

1. Thaw one $200-\mu \mathrm{L}$ aliquot of serum per subject/patient and distribute $50 \mu \mathrm{L}$ into three replicate 96-well Star PCR raised rim skirted plates. Change the location of samples across each replica plate. Also add $50 \mu \mathrm{L}$ of quality control (QC) serum at 6-12 random positions on each plate which are used to monitor assay reproducibility. Record all sample positions. Re-store plates at $-80^{\circ} \mathrm{C}$ and run each plate on 3 separate days.

2. Wash magnetic beads in $0.1 \%$ TFA solution and resuspend to a concentration of $50 \mathrm{mg} / \mathrm{mL}$ in an Eppendorf tube.

3. Carry out the following steps on a Genesis Freedom 200 liquid-handling work station (or similar) ${ }^{6}$ except where indicated.

4. Resuspend magnetic beads by pipetting up and down 10 times and transfer 5 $\mu \mathrm{L}$ to the wells of a 96-well plate containing the $50 \mu \mathrm{L}$ aliquots of serum.

5. Mix by pipetting up and down 10 times and allow to stand for 1 min.

6. Pull the beads to one side of the wells using the magnetic bead separator and allow beads to settle on the side for $30 \mathrm{sec}$.

\footnotetext{
${ }^{5}$ Blood collection requires informed consent from donors and studies require ethical approval from the relevant Committees on the Ethics of Human Research. Blood should be taken by a trained phlebotomist. Time of venepuncture should be recorded along with other subject/patient information relevant to the study, such as clinico-pathological features, treatment details, demographic data and any routine clinical assay results. Ideally, controls should be matched as closely as possible to cases. Ensure all samples are handled and processed identically (see Timms et al. 2010 for further information).

${ }^{6}$ Other robotic liquid-handling platforms can be employed, although the protocol would need to be adjusted for other platforms. For example, a CyBi ${ }^{\mathrm{TM}}$-Disk liquid handling robot (CyBio AG, Jena, Germany) with pre-packed C4 and C18 ZipTips (Millipore, Watford, UK) was used successfully in previous work (Tiss et al. 2007)
} 
7. Remove the supernatant and discard.

8. Add $200 \mu \mathrm{L}$ of wash solution ( $0.1 \%$ TFA) and pull the beads left to right 10 times using the magnetic bead separator and then allow to settle on one side for $30 \mathrm{sec}$.

9. Remove the wash solution and repeat the wash step.

10. Spin the beads to the bottom of the wells by centrifugation at $2,000 \mathrm{~g}$ for 2 min and remove remaining wash buffer.

11. Add $7 \mu \mathrm{L}$ of elution solvent consisting of $50 \%$ ACN in $0.1 \%$ TFA and mix by pipetting up and down 10 times. Leave the mixture to stand for $30 \mathrm{sec}$.

12. Transfer the 96-well plate to the magnetic bead separator and pull the beads to one side for $30 \mathrm{sec}$.

13. Add $35 \mu \mathrm{L}$ of pre-prepared CHCA matrix solution to each well and mix by pipetting up and down 5 times.

14. Spot volumes of $1 \mu \mathrm{L}$ of the eluate/matrix mix in quadruplicate onto a ground steel MALDI target plate and allow samples to dry at room temperature. ${ }^{7}$ This generates 12 spotting replicates per sample.

\subsubsection{MALDI-TOF MS data acquisition}

1. Externally calibrate the MALDI-TOF mass spectrometer ${ }^{8}$ in the linear positive ion mode using commercial peptide and protein calibration standards (in CHCA matrix) spotted onto the same target plate as the samples of interest. Use approximately $30 \mathrm{fmol}$ of peptide and $500 \mathrm{fmol}$ of protein standards per spot and use average masses for calibration.

2. Set the following automated irradiation program and data quality filtering using FlexControl's 'AutoXecute' function: each spectrum is the sum of 1,000 laser shots per spotted sample delivered to 10 different locations on the spot in 10 sets of 100 shots (at a laser frequency of $10 \mathrm{~Hz}$ ), after pre-irradiation

\footnotetext{
${ }^{7}$ Note that drying at relative humidity below $\sim 30-40 \%$ has been reported to be detrimental for MALDI MS (Tiss et al. 2007). Thus, ensure that the relative humidity is $\geq 35-40 \%$.

${ }^{8}$ The Ultraflex II MALDI-TOF/TOF mass spectrometer is equipped with a $337 \mathrm{~nm}$ nitrogen laser, a gridless ion source, delayed-extraction (DE), a high-resolution timed ion selector and a $2 \mathrm{GHz}$ digitizer. Other MALDI-TOF platforms can be used (see earlier footnote).
} 
with 10 shots at $5 \%$ higher laser power to improve spectral quality; set evaluation parameters so that only spectra (of 100 shots) containing at least one peak with a resolving power of greater than 300 and a signal-to-noise ratio $(\mathrm{S} / \mathrm{N})>10$ in the $\mathrm{m} / \mathrm{z}$ range of $700-4,000$ are accumulated.

3. Automatically acquire spectral profiles of the samples over a mass-to-charge $(\mathrm{m} / \mathrm{z})$ range of approximately $700-10,000$ in the linear positive ion mode under $25 \mathrm{kV}$ of ion acceleration, a delayed extraction (DE) potential difference of $1.4 \mathrm{kV}$, a lens potential of $5.9 \mathrm{kV}$ and high gating strength to deflect ions below $m / z$ 400. Apply DE (in our case at $80 \mathrm{~ns}$ ) to give appropriate time-lag focusing after each laser shot. For signal detection, the detector gain is typically set to 7.5 and the sample rate to $2 \mathrm{GS} / \mathrm{s}$.

4. For further analysis, only include data for an individual serum sample when at least 3 of the 4 spotting replicates had 1,000 summed shots in at least 2 of the 3 run replicates. Typical averaged spectra for case control serum samples are shown in Figure 2 with $~ 300$ aligned peaks detected across the dataset $(\mathrm{S} / \mathrm{N}>3.0)$. 
A
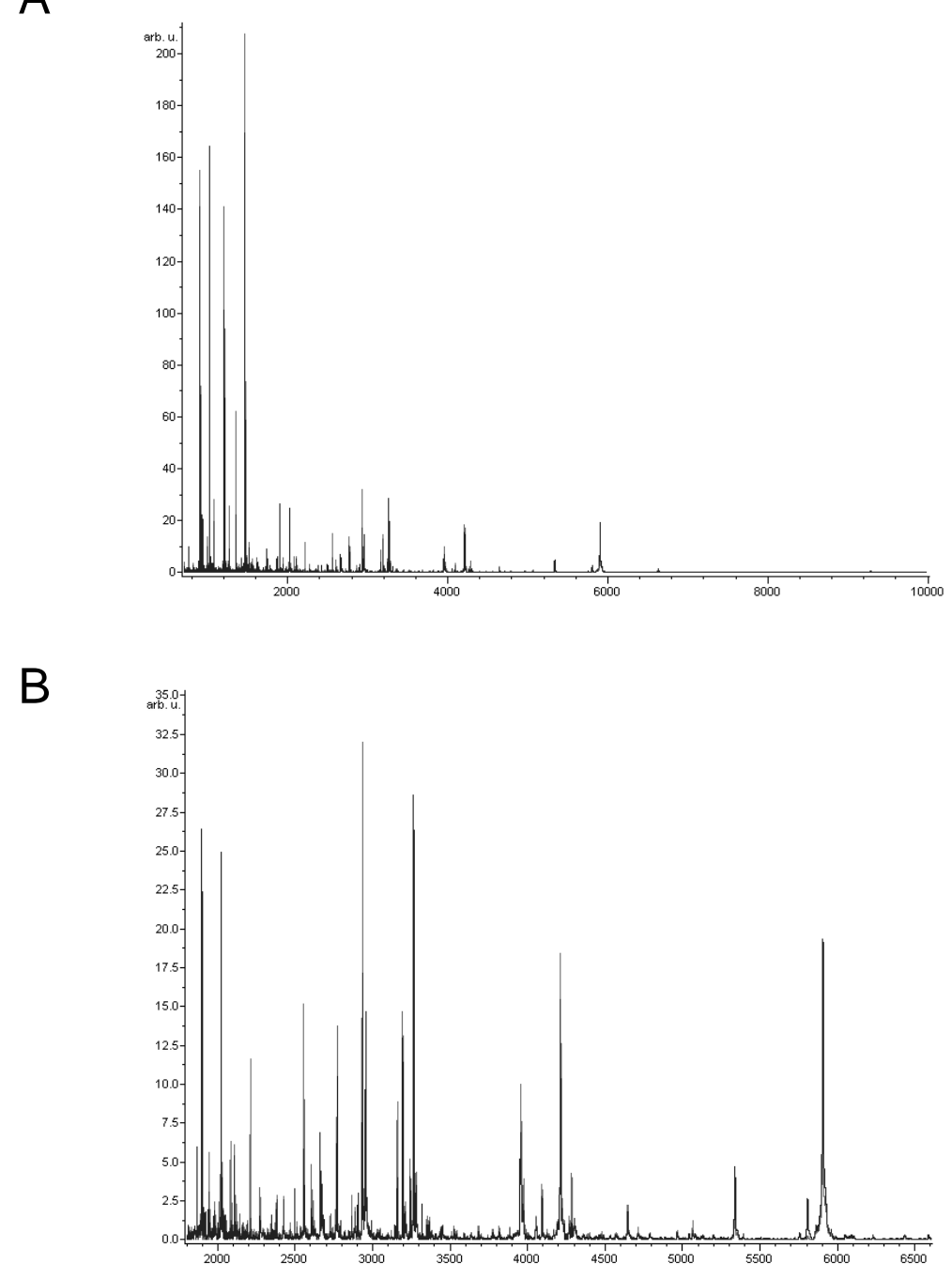

Figure 2: (A) Average MALDI-TOF MS spectra for case (dark grey) and control (light grey) serum samples over the full scan range of $m / z 700-10,000$. (B) Zoomed MS spectra over the $m / z$ range of $1,700-6,900$.

\subsubsection{Spectral data analysis ${ }^{9}$}

1. Use ClinProTools v2.2 software for processing spectral data as follows: apply smoothing by averaging the intensities within a 5-point width moving window; apply baseline subtraction using an algorithm based on finding the lowest points between dominant local intensity maxima within a particular mass window; apply normalisation by dividing the intensity of each data point in a

${ }^{9}$ See footnote to section 3.1.3. 
spectrum by the sum of all intensities in that spectrum; multiply intensities by a constant (e.g. $2 \times 10^{7}$ ) to give manageable output intensities; define peaks by identifying all local maxima in the spectra above a normalised intensity threshold of 0.2 and signal-to-noise ratio of 3 ; perform peak alignment to define common peaks using a mass window of $1,500 \mathrm{ppm}$.

2. Determine average peak areas and standard deviations for $Q C$ serum samples and for each sample group (case, control etc.). All aligned peaks from the QC samples can be used to determine intra-plate and inter-plate assay reproducibility which should be within $10 \%$ and $20 \%$, respectively (see Figure 3).

3. Determine distribution of peak areas and apply appropriate test of significance to define peaks, which discriminate between sample groups. Apply correction for multiple testing.

4. Split full dataset into training and test datasets. Construct multi-marker models (e.g. neural networks, support vector machines, genetic algorithms) from training data and determine classification performance on the test set, ideally in a blinded fashion. ${ }^{10}$

\footnotetext{
${ }^{10}$ Multi-marker model building and testing can be performed with the ClinProTools software. As before, other (classification) software can be used.
} 


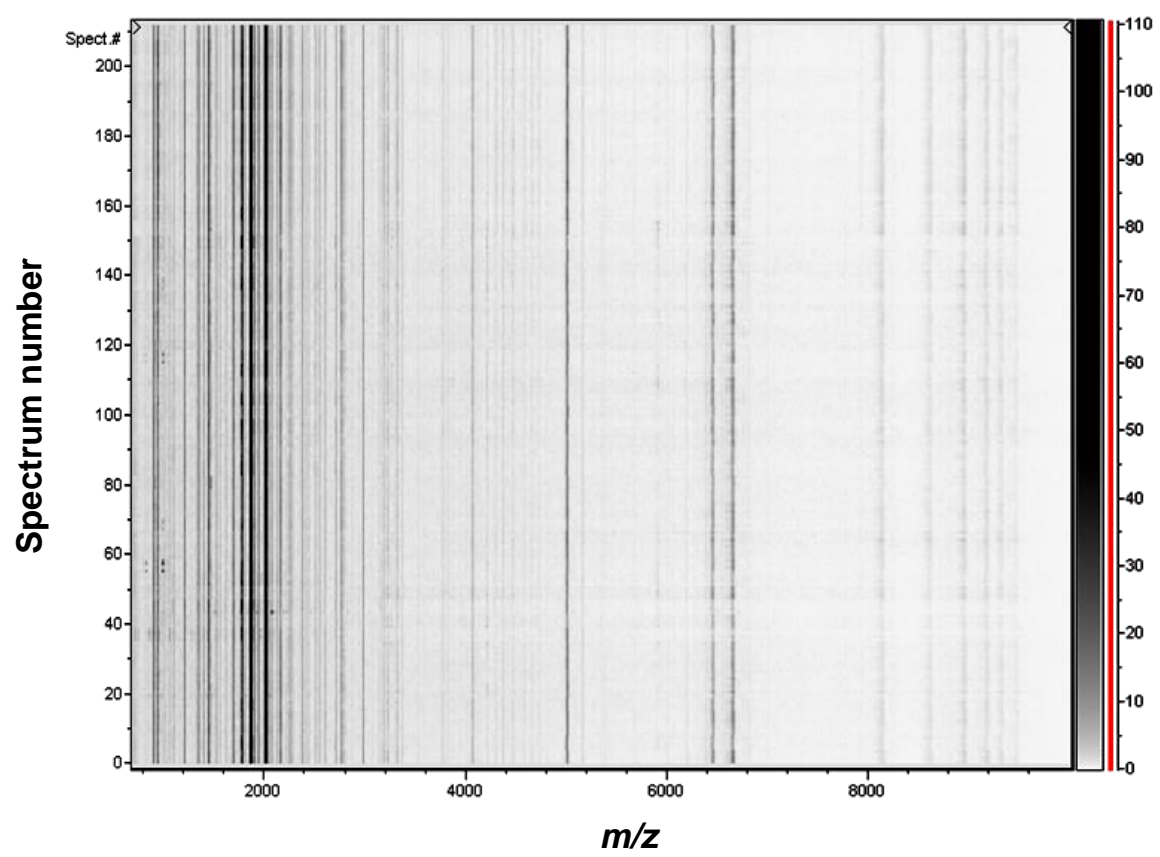

Figure 3: Aligned MS spectra of QC serum replicates in pseudo-gel view (3 replicate runs performed on different days with 12 samples per run each with 6 spotting replicates, i.e. total of 216 spectra). Using all peaks, intra-assay variation was $6.2 \% \pm 4.8$ and inter-assay variation was $13.9 \% \pm 7.6$.

\subsubsection{Identification of MALDI-TOF peaks by LC-MS/MS ${ }^{11}$}

1. Prepare a fresh C18-extracted sample from $50 \mu \mathrm{L}$ of serum without adding matrix solution (see Section 3.2.2.; up to step 12). Using an Ultimate 3000 LC system, inject $5 \mu \mathrm{L}$ of the extract from the autosampler onto a C18 PepMap guard column and wash for 3 min with $100 \%$ solvent $A$ at a flow rate of $25 \mu \mathrm{L} / \mathrm{min}$.

2. Switch to an analytical C18 PepMap nano LC column with $10 \%$ solvent $B$ and apply a linear gradient of $10-50 \%$ B over 90 min, then to $100 \%$ B over 3 min. Continue with $100 \%$ B for 20 min and then reduce to $10 \%$ B over 0.5 min and continue for a further $20 \mathrm{~min}$ to re-equilibrate the column for the next injection.

3. Operate the mass spectrometer (LTQ Orbitrap $X L$ ) in the data-dependent mode for automated switching between MS and MS/MS acquisition. Acquire

\footnotetext{
${ }^{11}$ See footnote to section 3.1.4.
} 
survey full scan MS spectra (from $\mathrm{m} / \mathrm{z} 400-2000$ ) in the orbitrap with a resolution of 60,000 at $\mathrm{m} / \mathrm{z} 400$. Select the 'top 6' most intense ions for CID. Select a target ion value of $1 \times 10^{6}$ and maximum scan time of $500 \mathrm{~ms}$ for the survey full scan in the orbitrap. Select target ion values of $1 \times 10^{4}$ and a scan time setting $150 \mathrm{~ms}$ for CID. Dynamically exclude ions selected for MS/MS for $60 \mathrm{sec}$. Enable the lock mass option for accurate mass measurement, using the polydimethylcyclosiloxane ion $(\mathrm{m} / \mathrm{z} 455.120025)$ as an internal calibrant.

4. Use initial precursor ion monitoring to identify masses matching the peaks of interest and then target these by mass and retention time in subsequent runs using high resolution MS/MS in the orbitrap.

5. Process raw spectra using Mascot Distiller and search against the human UniProtKB database. For searching, select 'no enzyme', set MS tolerance to $\pm 10 \mathrm{ppm}$ and MS/MS tolerance to $0.1 \mathrm{Da}$. Set oxidation (M), dehydration (Nterm $\mathrm{C}$ ) and deamidation (NQ) as variable modifications. Also search data in the same way using the 'de novo sequencing' function of Mascot Distiller. ${ }^{12}$

6. For larger peaks $(m / z>5,000)$, dry down the extracted sample in a SpeedVac, resuspend in sample buffer and resolve on a $10-20 \%$ gradient mini gel following the manufacturer's instructions. Stain the gel with InstantBlue and excise a gel piece in the molecular weight region of interest.

7. Destain the gel piece by shaking at room temperature for $30 \mathrm{~min}$ in $200 \mu \mathrm{L}$ of $50 \%$ methanol $/ 10 \%$ acetic acid and then wash the gel piece in $100 \mu \mathrm{L}$ of $100 \%$ ACN with shaking for 15 min. Extract polypeptides in $200 \mu \mathrm{L}$ of $50 \%$ formic acid, $25 \% \mathrm{ACN}, 15 \%$ isopropanol by vigorous shaking for $2 \mathrm{hrs}$ at room temperature. Centrifuge and recover the extract, dry down and resuspend in $0.1 \%$ formic acid. Analyse one fifth of this sample by MALDITOF MS (see Section 3.2.3.), to verify the presence of peaks of interest. ZipTip the remaining sample and analyse by LC-MS/MS as described above in this section, starting with the first step.

\footnotetext{
${ }^{12}$ If the identity of the peak of interest is still ambiguous, it may be necessary to carry out fractionation of the scaled-up extract. For a detailed protocol refer to (Tiss et al. 2010).
} 


\section{References}

Baggerly KA, Morris JS, Coombes KR (2004) Reproducibility of SELDI-TOF protein patterns in serum: comparing datasets from different experiments. Bioinformatics 20 (5):777-785

Baggerly KA, Morris JS, Edmonson SR, Coombes KR (2005) Signal in noise: evaluating reported reproducibility of serum proteomic tests for ovarian cancer. J Natl Cancer Inst 97 (4):307-309

Banks RE, Stanley AJ, Cairns DA, Barrett JH, Clarke P, Thompson D, Selby PJ (2005) Influences of blood sample processing on low-molecular-weight proteome identified by surface-enhanced laser desorption/ionization mass spectrometry. Clin Chem 51 (9):1637-1649

Conraux L, Pech C, Guerraoui H, Loyaux D, Ferrara P, Guillemot JC, Meininger V, Pradat PF, Salachas F, Bruneteau G, Le Forestier N, Lacomblez L (2013) Plasma peptide biomarker discovery for amyotrophic lateral sclerosis by MALDITOF mass spectrometry profiling. PLoS One 8 (11):e79733. doi:10.1371/journal.pone.0079733

Davis MT, Auger PL, Patterson SD (2010) Cancer biomarker discovery via low molecular weight serum profiling--are we following circular paths? Clin Chem 56 (2):244-247. doi:10.1373/clinchem.2009.127951

Diamandis EP (2004a) Analysis of serum proteomic patterns for early cancer diagnosis: drawing attention to potential problems. J Natl Cancer Inst 96 (5):353-356

Diamandis EP (2004b) Mass spectrometry as a diagnostic and a cancer biomarker discovery tool: opportunities and potential limitations. Mol Cell Proteomics 3 (4):367-378

Diamandis EP (2006) Serum proteomic profiling by matrix-assisted laser desorptionionization time-of-flight mass spectrometry for cancer diagnosis: next steps. Cancer Res 66 (11):5540-5541

Geho DH, Liotta LA, Petricoin EF, Zhao W, Araujo RP (2006) The amplified peptidome: the new treasure chest of candidate biomarkers. Curr Opin Chem Biol 10 (1):50-55. doi:10.1016/j.cbpa.2006.01.008

Hortin GL (2006) The MALDI-TOF mass spectrometric view of the plasma proteome and peptidome. Clin Chem 52 (7):1223-1237

Karsan A, Eigl BJ, Flibotte S, Gelmon K, Switzer P, Hassell P, Harrison D, Law J, Hayes M, Stillwell M, Xiao Z, Conrads TP, Veenstra T (2005) Analytical and preanalytical biases in serum proteomic pattern analysis for breast cancer diagnosis. Clin Chem 51 (8):1525-1528

Li L, Li J, Jin H, Shang L, Li B, Wei F, Liu Q (2012) Detection of Leishmania donovani infection using magnetic beads-based serum peptide profiling by MALDI-TOF MS in mice model. Parasitol Res 110 (3):1287-1290. doi:10.1007/s00436-011-2604-0 
Liotta LA, Petricoin EF (2006) Serum peptidome for cancer detection: spinning biologic trash into diagnostic gold. J Clin Invest 116 (1):26-30. doi:10.1172/JCl27467

Lu J, Huang Y, Wang Y, Li Y, Zhang Y, Wu J, Zhao F, Meng S, Yu X, Ma Q, Song $M$, Chang N, Bittles AH, Wang W (2012) Profiling plasma peptides for the identification of potential ageing biomarkers in Chinese Han adults. PLoS One 7 (7):e39726. doi:10.1371/journal.pone.0039726

Petricoin EF, Belluco C, Araujo RP, Liotta LA (2006) The blood peptidome: a higher dimension of information content for cancer biomarker discovery. Nat Rev Cancer 6 (12):961-967. doi:nrc2011 [pii] 10.1038/nrc2011

Pietrowska M, Marczak L, Polanska J, Behrendt K, Nowicka E, Walaszczyk A, Chmura A, Deja R, Stobiecki M, Polanski A, Tarnawski R, Widlak P (2009) Mass spectrometry-based serum proteome pattern analysis in molecular diagnostics of early stage breast cancer. J Transl Med 7:60. doi:10.1186/14795876-7-60

Sandanayake NS, Camuzeaux S, Sinclair J, Blyuss O, Andreola F, Chapman MH, Webster GJ, Smith RC, Timms JF, Pereira SP (2014) Identification of potential serum peptide biomarkers of biliary tract cancer using MALDI MS profiling. BMC Clin Pathol 14 (1):7. doi:10.1186/1472-6890-14-7

Taguchi F, Solomon B, Gregorc V, Roder H, Gray R, Kasahara K, Nishio M, Brahmer J, Spreafico A, Ludovini V, Massion PP, Dziadziuszko R, Schiller J, Grigorieva J, Tsypin M, Hunsucker SW, Caprioli R, Duncan MW, Hirsch FR, Bunn PA, Jr., Carbone DP (2007) Mass spectrometry to classify non-small-cell lung cancer patients for clinical outcome after treatment with epidermal growth factor receptor tyrosine kinase inhibitors: a multicohort cross-institutional study. J Natl Cancer Inst 99 (11):838-846. doi:10.1093/jnci/djk195

Terracciano R, Preiano M, Palladino GP, Carpagnano GE, Barbaro MP, Pelaia G, Savino R, Maselli R (2011) Peptidome profiling of induced sputum by mesoporous silica beads and MALDI-TOF MS for non-invasive biomarker discovery of chronic inflammatory lung diseases. Proteomics 11 (16):3402-3414. doi:10.1002/pmic.201000828

Teunissen CE, Koel-Simmelink MJ, Pham TV, Knol JC, Khalil M, Trentini A, Killestein J, Nielsen J, Vrenken H, Popescu V, Dijkstra CD, Jimenez CR (2011) Identification of biomarkers for diagnosis and progression of MS by MALDI-TOF mass spectrometry. Mult Scler 17 (7):838-850. doi:10.1177/1352458511399614

Timms JF, Arslan-Low E, Gentry-Maharaj A, Luo Z, T'Jampens D, Podust VN, Ford J, Fung ET, Gammerman A, Jacobs I, Menon U (2007) Preanalytic influence of sample handling on SELDI-TOF serum protein profiles. Clin Chem 53 (4):645656

Timms JF, Cramer R, Camuzeaux S, Tiss A, Smith C, Burford B, Nouretdinov I, Devetyarov D, Gentry-Maharaj A, Ford J, Luo Z, Gammerman A, Menon U, Jacobs I (2010) Peptides generated ex vivo from serum proteins by tumor- 
specific exopeptidases are not useful biomarkers in ovarian cancer. Clin Chem 56 (2):262-271

Timms JF, Menon U, Devetyarov D, Tiss A, Camuzeaux S, McCurrie K, Nouretdinov I, Burford B, Smith C, Gentry-Maharaj A, Hallett R, Ford J, Luo Z, Vovk V, Gammerman A, Cramer R, Jacobs I (2011) Early detection of ovarian cancer in samples pre-diagnosis using CA125 and MALDI-MS peaks. Cancer Genomics Proteomics 8 (6):289-305

Tiss A, Smith C, Camuzeaux S, Kabir M, Gayther S, Menon U, Waterfield M, Timms JF, Jacobs I, Cramer R (2007) Serum peptide profiling using MALDI mass spectrometry: avoiding the pitfalls of coated magnetic beads using wellestablished ZipTip technology. Proteomics 7 Suppl 1:77-89

Tiss A, Smith C, Menon U, Jacobs I, Timms JF, Cramer R (2010) A wellcharacterised peak identification list of MALDI MS profile peaks for human blood serum. Proteomics 10 (18):3388-3392

Vafadar-Isfahani B, Laversin SA, Ahmad M, Ball G, Coveney C, Lemetre C, Kathleen Miles A, van Schalkwyk G, Rees R, Matharoo-Ball B (2010) Serum biomarkers which correlate with failure to respond to immunotherapy and tumor progression in a murine colorectal cancer model. Proteomics Clin Appl 4 (89):682-696. doi:10.1002/prca.200900218

Villanueva J, Lawlor K, Toledo-Crow R, Tempst P (2006a) Automated serum peptide profiling. Nat Protoc 1 (2):880-891

Villanueva J, Martorella AJ, Lawlor K, Philip J, Fleisher M, Robbins RJ, Tempst P (2006b) Serum Peptidome Patterns That Distinguish Metastatic Thyroid Carcinoma from Cancer-free Controls Are Unbiased by Gender and Age. Mol Cell Proteomics 5 (10):1840-1852

Villanueva J, Shaffer DR, Philip J, Chaparro CA, Erdjument-Bromage H, Olshen AB, Fleisher M, Lilja H, Brogi E, Boyd J, Sanchez-Carbayo M, Holland EC, CordonCardo C, Scher HI, Tempst P (2006c) Differential exoprotease activities confer tumor-specific serum peptidome patterns. J Clin Invest 116 (1):271-284

Xiao D, Meng FL, He LH, Gu YX, Zhang JZ (2011) Analysis of the urinary peptidome associated with Helicobacter pylori infection. World J Gastroenterol 17 (5):618-624. doi:10.3748/wjg.v17.i5.618 


\section{Acknowledgements}

This work was funded by MRC grant G0301107 and was supported by the National

Institute for Health Research University College London Hospitals Biomedical Research Centre. 